\title{
Diabetes, the kidney and the heart-A tale of metabolic mayhem
}

\section{Opinion}

The epidemic of diabetes in the modern world continues at a very rapid pace. In addition, with this rising need is the evolution of new therapies for the management of diabetes. Due to large-scale cardiovascular outcomes studies, notable end organ benefits have been demonstrated with some of these newer agents, particularly focusing on obesity, cardiovascular health/mortality, the kidney, and the liver.

As we are aware, cardiovascular complications of are the main causes of mortality and in diabetes. Patients with diabetes or are 4 times more likely to suffer with coronary artery disease and stroke. ${ }^{1}$ It is also notable that for every $1 \%$ increase in hemoglobin A1c, the risk of stroke has is increased by as much as $30 \%{ }^{2}$ Two thirds of death in patients with diabetes are attributed to cardiovascular disease, ${ }^{3}$ which means that diabetics have a 2-6 time lifetime higher risk of mortality related to cardiovascular events. ${ }^{4}$ Heart failure, regardless if related to heart failure with preserved ejection fraction or reduced ejection fraction, is also prevalent in this population although heart failure with preserved ejection fraction tends to predominate. ${ }^{5}$ Numerous risk factors have also been explored in terms of cardiovascular risk, specifically, cholesterol, cigarette smoking status, and systolic blood pressure. It is interesting to note that age adjusted cardiovascular death risk per 10,000 person years escalates at a near hyperbolic response in patients with multiple of these aforementioned risk factors after suffering with diabetes over 10years. ${ }^{6}$ Likewise, the converse may apply where intensive treatment with reduction of risk factors mentioned lit led to significant reduction in cumulative death and cardiovascular events as was demonstrated in the historic STENO 2 trial. $^{7}$

While we've learned to recognize that duration of diabetes increases his the risk of cardiovascular disease leadsing to by an exponential degree after 10years of suffering with diabetes, ${ }^{8,9}$ it is equally important to recognize that life expectancy may be reduced by as much as 6.2 years just by having diabetes, 12.8 years if you've had a diabetes and myocardial infarction, 15.1years respectively if you've had diabetes, myocardial infarction and stroke. ${ }^{10,11}$

Of antidiabetic therapies currently available, as listed in the American Association of Clinical Endocrinologists algorithm and the consensus statement from the American Diabetes Association, the GLP-1 receptor agonists and SGL T2 inhibitors, for the most part, have demonstrated either cardiovascular safety or reduction in major adverse cardiovascular events. More recent guidance issued by the ADA recommends utilization of these agents regardless of where the hemoglobin A1c may be because of its cardiovascular benefit. However, In terms of reduction in major adverse cardiovascular events, Liraglutide, semaglutide as a weekly injection and Dulaglutide have demonstrated reduction in major adverse cardiovascular events and as a result, has we gained and FDA indication for such cardiovascular risk reduction. Likewise, Empagliflozin has been recognized for his its reduction in cardiovascular death, and heart failure, with canagliflozin having been recognized for reductions in major adverse cardiovascular events, as well as beneficial effects on kidney function.
Volume I4 Issue I - 202I

\author{
Javier Morales,' 'Merces Assumpcao-Morales ${ }^{2}$ \\ 'Advanced Internal Medicine Group, USA \\ ${ }^{2}$ Associate Clinical Professor of Medicine, New York \\ UniversityWinthrop University Hospital, USA
}

Correspondence: Javier Morales, Associate Clinical Professor of Medicine, Donald and Barbara Zucker School of Medicine at Hofstra/Northwell University,Vice President, Advanced Interna Medicine Group, PC, 2200 Northern Blvd., Suite 133, East Hills, New York, USA, Tel 516-637-4947, 516-637-4947, Fax 516-352 7348,Email saxodo@gmail.com

Received: January 15, 202I | Published: February 24, 2021

Dapagliflozin has also gained the indication for reduction in heart failure hospitalizations I diabetics. ${ }^{12}$

Diabetic kidney disease is also very common in the clinical course of patients with type 2 diabetes. The presence of protein in the urine indicates glomerular injury and as a result, use of urine albumin/ creatinine ratio tends to crudely correlate with a standard 24-hour urine collection for protein. However, because there are so many different causes of progressive kidney disease, the diagnosis of kidney disease attributed to diabetes is defined on the basis of the presence of albuminuria (UACr $>30 \mathrm{mg} / \mathrm{g}$ or UACr ratio of $30-299 \mathrm{mg} / \mathrm{g}$ with concomitant diabetic retinopathy and/or having had type 1 diabetes for greater than 10years in duration) or reduced kidney function as defined by an eGFR $<60 \mathrm{~mL} / \mathrm{min} / 1.73 \mathrm{~m} 2 .{ }^{13}$ We also have learned to recognize that compared to healthy individuals, diabetic kidney disease will shortened life expectancy by as much is 16years. ${ }^{14,15}$ The association of diabetic kidney disease promoting cardiovascular diseases is largely driven by such comorbidities, such as endothelial dysfunction, inflammation and oxidative stress, hypercoagulability, dyslipidemia, hypertension, which all worsen in the presence of such kidney disease. ${ }^{16-18}$ It also has been demonstrated that appropriate intervention designed to arrest the progression of kidney disease would lessen cardiovascular events. ${ }^{15}$ The urgency of appropriate intervention can be determined by using the heat map that was developed by KDIGO. ${ }^{19}$

Review of most of the major cardiovascular outcomes studies completed have demonstrated a significant beneficial effect on delaying the progression of chronic kidney disease that favors the use of GLP-1 receptor agonists as well as SGL T2 receptor inhibitors.

As a result of such data, I offered through the ADA recommends the use of SGL T2 inhibitors for reducing heart failure and or chronic kidney disease progression if eGFR allows, otherwise, a GLP-1 receptor agonist with proven cardiovascular benefit may be utilized. 
Traditionally, we have been using AceCE inhibitors and angiotensin receptor blockers to help reduce proteinuria by reducing intraglomerular pressure via efferent arteriolar relaxation. However, we also have learned that there are various inflammatory markers which may be responsible for worsening kidney function through various mechanisms leading to fibrosis. The use of mineralocorticoid receptor antagonists have a demonstrable effect on left ventricular remodeling and inflammatory markers, however, limited by the potentials of precipitating serum hyperkalemia. However, the evolution of nonsteroidal mineralocorticoid receptor antagonist have offered the same reduction inflammation and in clinical trials performed, less hyperkalemia demonstrated especially with concomitant use of an ACE inhibitor or angiotensin receptor blocker. In the fall of 2020, such and nonsteroidal mineralocorticoid receptor antagonist, Finerenone had completed and reported a very large was studied in a trial in which 5734 patients were randomized to receive Finerenone at a dose of $10 \mathrm{mg}$ or $20 \mathrm{mg}$ daily versus placebo. The primary endpoint of the trial was time to kidney failure, sustained greater than or equal to $40 \%$ decrease in eGFR from baseline or renal death, and the secondary composite endpoint looking at that included cardiovascular death, nonfatal MI, non-fatal stroke or hospitalization for heart failure. After 36 months, there was a very rapid and sustainable reduction in urine albumin creatinine ratio on the magnitude order of $31 \%$, with the primary endpoint as described above having been reduced by $18 \%$, and satisfied ying statistical significance with the P value of 0.0014 . These data were reported in the fall of 2020.

The secondary endpoint, which was the 4-point Mace ACE described above demonstrated a $14 \%$ reduction satisfiedying statistical significance with the $\mathrm{P}$ value of $0.0339 .{ }^{20} \mathrm{Changes}$ in serum potassium, as depicted in this clinical trial between both groups, was $0.23 \mathrm{mmol}$ per liter at the fourth month data collection point, and the lower measured serum potassium continued to persist throughout the course of the trial.

The association of between the kidney and the heart diseasewith an associated increase in mortality has always been known acknowledged and increasingly better understood because of the identified metabolically apparent disease that could be abberancies created by such associated with worsening renal dysfunction. Appropriate medication selection that may impact the natural history of chronic kidney disease may prove superfluous in terms of reducing overall cardiovascular events and mortality. Now that we have traditionally been using AceCE inhibitors and angiotensin receptor blockers, and gaineding confidence using GLP-1 receptor agonists and SGL T2 receptor inhibitors antagonists, it appears that the concomitant administration of nonsteroidal mineralocorticoid receptor antagonists, such as Finerenone, may prove a useful Aally in the armamentarium of further reducing cardiovascular risk, preserving renal function and perhaps beyond.

\section{Acknowledgements}

None.

\section{Conflicts of interest}

The rest of the authors declare do not have conflicts of interest.

\section{Funding}

None.

\section{References}

1. Lüscher TF, Creager MA, Beckman JA, et al. Diabetes and Vascular Disease Pathophysiology, Clinical Consequences, and Medical Therapy: Part II. Circulation. 2003;108:1655-1661.

2. Mombach JCM, Vendrusculo B, Bugs CA. A Model for p38MAPKInduced Astrocyte Senescence. PLoS One. 2015;10:1-12.

3. Low Wang CC, Hess CN, Hiatt WR, et al. Clinical Update: Cardiovascular Disease in Diabetes MellitusAtherosclerotic Cardiovascular Disease and Heart Failure in Type 2 Diabetes Mellitus - Mechanisms, Management, and Clinical Considerations. Circulation. 2016;133:2459-2502.

4. Fihn SD, Gardin GM, Abrams J, et al. 2012 ACCF/AHA/ACP/AATS/ PCNA/SCAI/STS Guideline for the diagnosis and management of patients with stable ischemic heart disease: a report of the American College of Cardiology Foundation/American Heart Association Task Force on Practice Guidelines, and the American College of Physicians, American Association for Thoracic Surgery, Preventive Cardiovascular Nurses Association, Society for Cardiovascular Angiography and Interventions, and Society of Thoracic Surgeons. J Am Coll Cardiol. 2012;60(24):e44-e164.

5. Nichols GA, Gullion CM, Koro CE, et al. The Incidence of Congestive Heart Failure in Type 2 Diabetes. Diabetes Care. 2004;27:1879-1884.

6. Stamler J, Vaccaro O, Neaton JD, et al. Diabetes, other risk factors, and 12-yr cardiovascular mortality for men screened in the Multiple Risk Factor Intervention Trial. Diabetes Care. 1993;16(2):434-444.

7. Gaede P, Lund-Andersen H, Parving HH, et al. Effect of a multifactorial intervention on mortality in type 2 diabetes. $N$ Engl J Med. 2008;358:580-591.

8. Huo X, Gao L, Guo L, et al. Risk of non-fatal cardiovascular diseases in early-onset versus late-onset type 2 diabetes in China: a cross-sectional study. Lancet Diabetes Endocrinol. 2016;4(2):115-124.

9. Juutilainen A, Lehto S, Rönnemaa T, et al. Similarity of the impact of type 1 and type 2 diabetes on cardiovascular mortality in middle-aged subjects. Diabetes Care. 2008;31:714-719.

10. The Emerging Risk Factors Collaboration, Kaptoge S, Wormser D. Association of Cardiometabolic Multimorbidity With Mortality. JAMA. 2015;314(1):52-60.

11. Murphy SL, Xu J, Kochanek KD, et al. Mortality in the United States, 2017. NCHS Data Brief No.328 Hyattsville, MD. National Center for Health Sciences. 2018

12. Morales J, Assumpcao-Morales M. The use of SGL T2 inhibitors and GLP-1 receptor agonists, a worthwhile physiologic combination in managing type 2 diabetes while reducing cardiovascular risk. J Cardiol Curr Res. 2019;12(5):104-110.

13. National Kidney Foundation, KDOQI Guidelines Executive Summary. 2017.

14. Alicic RZ, Rooney MT, Tuttle KR, et al. Diabetic Kidney Disease: Challenges, Progress, and Possibilities. Clin J Am Soc Nephrol. 2017;12(12):2032-2045.

15. Wen $\mathrm{CP}$, Chang $\mathrm{CH}$, Tsai MK, et al. Diabetes with early kidney involvement may shorten life expectancy by 16 years. Kidney Int. 2017:92(2):388-396.

16. Go AS, Chertow GM, Fan D, et al. Chronic kidney disease and the risks of death, cardiovascular events, and hospitalization. $N$ Eng J Med. 2004;351(13):1296-1305.

17. Sasso FC, Chiodini P, Carbonara O, et al. High cardiovascular risk in patients with Type 2 diabetic nephropathy: the predictive role of albuminuria and glomerular filtration rate. The NID-2 Prospective Cohort Study. Nephrol Dial Transplant. 2012;27(6):2269-2274. 
18. Palsson R, Patel UD. Cardiovascular Complications of Diabetic Kidney Disease. Adv Chronic Kidney Dis. 2014;21(3):273-280.

19. Tuttle KR, Bakris GL, Bilous RW, et al. Diabetic Kidney Disease: A Report from an ADA Consensus Conference. Diabetes Care. 2014;37:2864-2883.
20. Improving Global Outcomes (KDIGO) CKD Work Group. KDIGO 2012 Clinical Practice Guideline for the Evaluation and Management of Chronic Kidney Disease. Kidney Int Suppls. 2013;3:1-150.

21. Bakris GL, Agarwal R, Anker SD, et al. Effect of Finerenone on Chronic Kidney Disease Outcomes in Type 2 Diabetes. $N$ Engl $J$ Med. 2020;383(23):2219-2229. 